\title{
Retraction Note: SARS-CoV-19's actual initial cases in Wuhan, China and the impact of different interventions and imports in the pandemic
}

Xiaochuan Li

No.12, Ganshui Rd., Xiangfang District, Harbin City, Heilongjiang Prov, China

Published online: 24 March 2021

Retracted article: E3S Web of Conferences 245, 03047 (2021)

https://doi.org/10.1051/e3sconf/202124503047

We take a zero tolerance to any situation where fraudulent research is published in our journals. As a result, this article has been retracted by the Publisher because it is suspected to be a nonsensical publication computer-generated by the program SCIgen. 